\title{
GENFIRE: from Precisely Localizing Single Atoms in Materials to High Resolution 3D Imaging of Cellular Structures
}

Jianwei Miao $^{1 *}$, Alan Pryor Jr. ${ }^{1}$, Yongsoo Yang ${ }^{1}$, Arjun Rana ${ }^{1}$, Marcus Gallagher-Jones ${ }^{2}$, Jihan Zhou ${ }^{1}$, Yuan Hung Lo ${ }^{1,3}$, Jose A. Rodriguez ${ }^{2}$ and Wah Chiu ${ }^{4}$

${ }^{1}$ Department of Physics and Astronomy and California NanoSystems Institute, University of California Los Angeles, California 90095, USA. ${ }^{2}$ Department of Chemistry \& Biochemistry, UCLA-DOE Institute of Genomics and Proteomics, Los Angeles, California 90095-1570, USA. ${ }^{3}$ Department of Bioengineering, University of California, Los Angeles, CA 90095, USA. ${ }^{4}$ SLAC National Accelerator Laboratory and Departments of Bioengineering, Microbiology and Immunology, Stanford University, Stanford, California, 94304, USA.

Tomography has made a radical impact on diverse fields ranging from the study of 3D atomic arrangements in matter to the study of human health in medicine. Despite its very diverse applications, the core of tomography remains the same, that is, a mathematical method must be implemented to reconstruct the 3D structure of an object from a number of $2 \mathrm{D}$ projections. Here, we present the mathematical implementation of a tomographic algorithm, termed GENeralized Fourier Iterative REconstruction (GENFIRE) [1], for high-resolution 3D reconstruction from a limited number of 2D projections. GENFIRE, like equal slope tomography [2-6], first assembles a 3D Fourier grid with oversampling and then iterates between real and reciprocal space to search for a global solution that is concurrently consistent with the measured data and general physical constraints. The algorithm requires minimal human intervention and also incorporates angular refinement to reduce the tilt angle error. We demonstrate that GENFIRE can produce superior results relative to several other popular tomographic reconstruction techniques through numerical simulations and by experimentally determining the 3D atomic positions in materials and imaging frozen-hydrated cellular structures at high resolution [1,7]. Equipped with a graphical user interface, GENFIRE is freely available from our website and is expected to find broad applications across different disciplines (http://genfire-em.com).

\section{References:}

1. Pryor, A. Jr. et al. GENFIRE: A generalized Fourier iterative reconstruction algorithm for highresolution 3D imaging. Sci. Rep. 7, 10409 (2017).

2. Miao, J., Föster, F. \& Levi, O. Equally sloped tomography with oversampling reconstruction. Phys. Rev. B 72, 052103 (2005).

3. E. Lee et al. Radiation Dose Reduction and Image Enhancement in Biological Imaging through Equally Sloped Tomography. J. Struct. Biol. 164, 221-227 (2008).

4. Scott, M. C. et al. Electron tomography at 2.4-angstrom resolution. Nature 483, 444-447 (2012).

5. Miao, J., Ercius, P. and Billinge, S. J. L. Atomic electron tomography: 3D structures without crystals. Science 353, aaf2157 (2016).

6. $\mathrm{Xu}, \mathrm{R}$. et al. Three-Dimensional Coordinates of Individual Atoms in Materials Revealed by Electron Tomography. Nature Mater. 14, 1099-1103 (2015).

7. Yang, Y. et al. Deciphering chemical order/disorder and material properties at the single-atom level. Nature 542, 75-79 (2017).

8. This work was supported by STROBE: A National Science Foundation Science \& Technology Center under Grant No. DMR 1548924, and the Office of Basic Energy Sciences of the U.S. Department of Energy (Grant No. DE-SC0010378). 

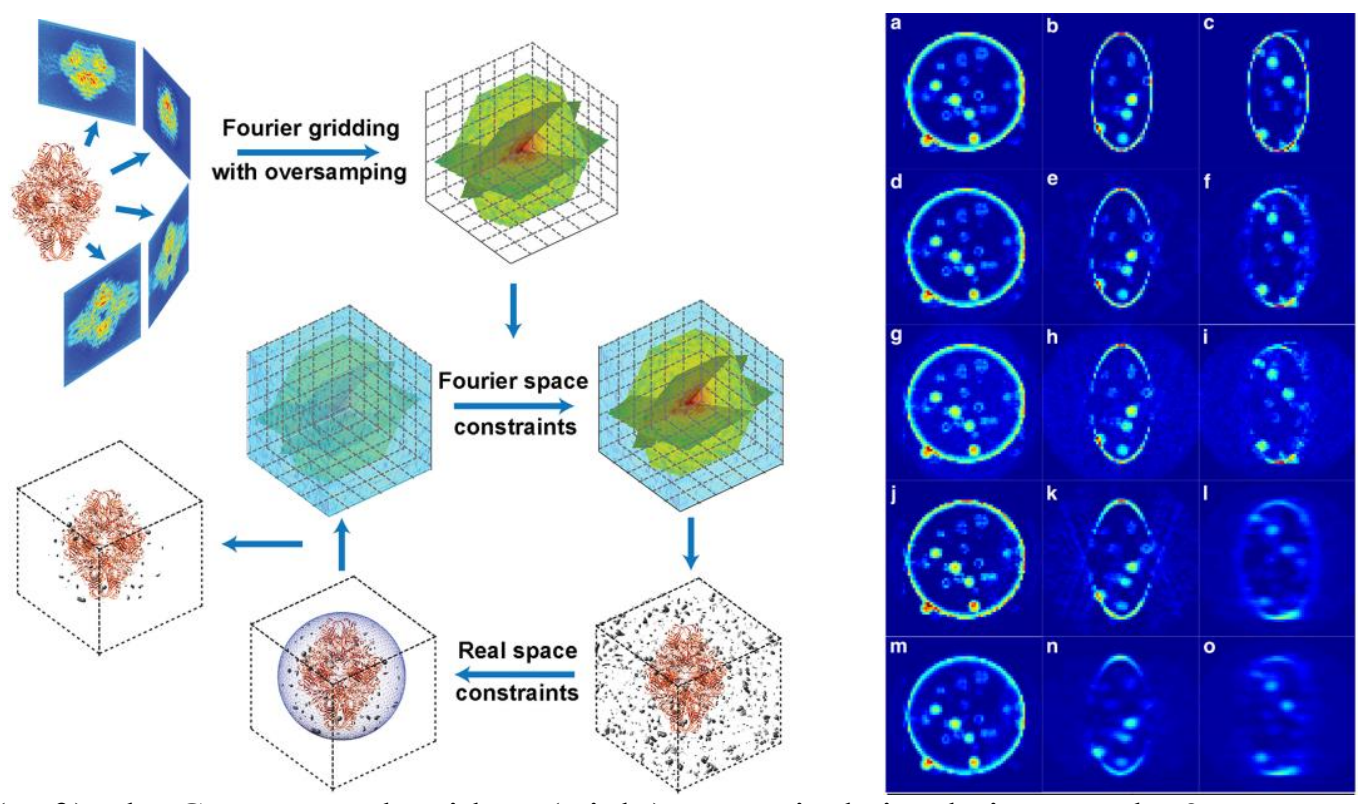

Figure 1. (Left) The GENFIRE algorithm. (Right) Numerical simulations on the 3D reconstruction of a $64 \times 64 \times 64$ voxel vesicle model from 71 noisy projections using GENFIRE, EST, FBP and SIRT. $(\mathrm{a}-\mathrm{c})$ Three 10-voxel-thick central slices of the vesicle model in the XY, ZX and ZY planes, respectively. The corresponding three reconstructed slices with GENFIRE $(\mathrm{d}-\mathrm{f})$, EST $(\mathrm{g}-\mathrm{i}), \mathrm{FBP}(\mathrm{j}-\mathrm{l})$, and SIRT $(\mathrm{m}-\mathrm{o})$, where the missing wedge axis is along the $\mathrm{z}$-axis.

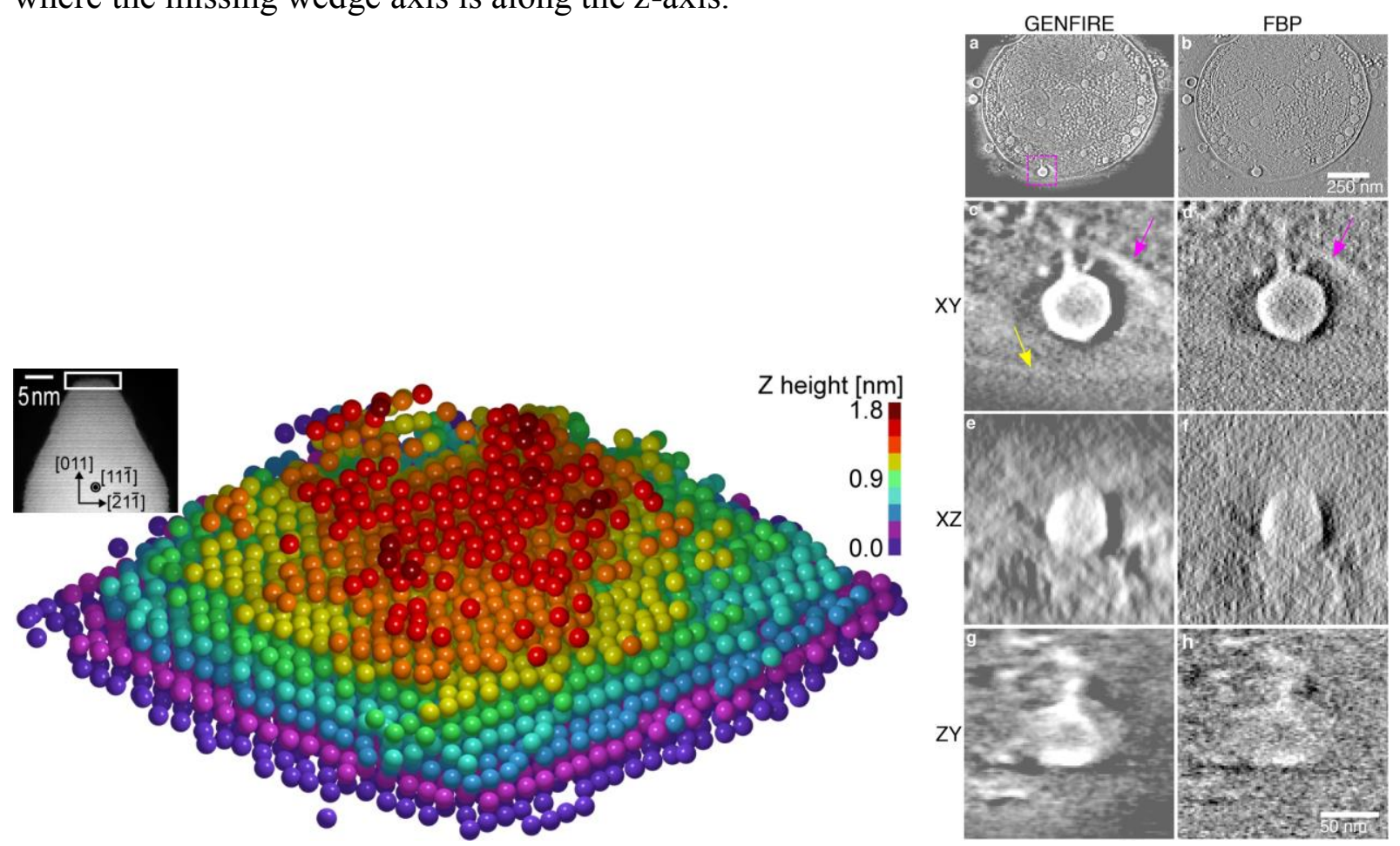

Figure 2. (Left) 3D positions of individual atoms in a tungsten needle sample revealed by atomic electron tomography with a 3D precision of 19 picometers. (Right) 3D structure of a frozen-hydrated marine cyanobacterium. (a, b) 5.4-nm-thick slices of the cell in the XY plane reconstructed by GENFIRE and FBP, respectively, Magnified views of the penetration of a cyanophage for the GENFIRE and FBP reconstructions in the XY (c, d), XZ (e, f), and ZY $(\mathrm{g}, \mathrm{h})$ planes, respectively. 